\title{
An innovative method to assess clinical reasoning skills: Clinical reasoning tests in the second national medical science Olympiad in Iran
}

Mitra Amini ${ }^{1}$, Mohsen Moghadami ${ }^{1 *}$, Javad Kojuri ${ }^{1}$, Hamidreza Abbasi ${ }^{1}$, Ali Arhami Doolat Abadi ${ }^{2}$, Nezar Ali Molaee ${ }^{3}$, Elham Pishbin ${ }^{4}$, Hamid Reza Javadzade ${ }^{5}$, Vahid Monsef Kasmaee ${ }^{6}$, Hasan Vakili ${ }^{7}$, Mohamad Ali Reis Sadat ${ }^{8}$, Roghaye Akbari ${ }^{7}$, Bita Omidvar ${ }^{9}$, Afshin Shafaghi $^{6}$, Marzie Dehbozorgian ${ }^{1}$, Mohammad Morad Jafari ${ }^{1}$, Alireza Monajemi ${ }^{10}$, Kamran Soltani Arabshahi ${ }^{11}$, Peyman Adibi ${ }^{12}$ and Bernard Charlin ${ }^{13}$

\begin{abstract}
Background: Clinical reasoning plays a major role in the ability of doctors to make a diagnosis and reach treatment decisions. This paper describes the use of four clinical reasoning tests in the second National Medical Science Olympiad in Iran: key features (KF), script concordance (SCT), clinical reasoning problems (CRP) and comprehensive integrative puzzles (CIP). The purpose of the study was to design a multi instrument for multiple roles approach in clinical reasoning field based on the theoretical framework, KF was used to measure data gathering, CRP was used to measure hypothesis formation, SCT and CIP were used to measure hypothesis evaluation and investigating the combined use of these tests in the Olympiad. A bank of clinical reasoning test items was developed for emergency medicine by a scientific expert committee representing all the medical schools in the country. These items were pretested by a reference group and the results were analyzed to select items that could be omitted. Then 135 top-ranked medical students from 45 medical universities in Iran participated in the clinical domain of the Olympiad. The reliability of each test was calculated by Cronbach's alpha. Item difficulty and the correlation between each item and the total score were measured. The correlation between the students' final grade and each of the clinical reasoning tests was calculated, as was the correlation between final grades and another measure of knowledge, i.e., the students' grade point average.

Results: The combined reliability for all four clinical reasoning tests was 0.91 . Of the four clinical reasoning tests we compared, reliability was highest for CIP (0.91). The reliability was 0.83 for KF, 0.78 for SCT and 0.71 for CRP. Most of the tests had an acceptable item difficulty level between 0.2 and 0.8 . The correlation between the score for each item and the total test score for each of the four tests was positive. The correlations between scores for each test and total score were highest for KF and CIP. The correlation between scores for each test and grade point average was low to intermediate for all four of the tests.
\end{abstract}

Conclusion: The combination of these four clinical reasoning tests is a reliable evaluation tool that can be implemented to assess clinical reasoning skills in talented undergraduate medical students, however these data may not generalizable to whole medical students population. The CIP and KF tests showed the greatest potential to measure clinical reasoning skills. Grade point averages did not necessarily predict performance in the clinical domain of the national competitive examination for medical school students.

\footnotetext{
* Correspondence: moghadami@sums.ac.ir

'Education Development and Research Center, Shiraz University of Medical

Sciences, Shiraz, Iran

Full list of author information is available at the end of the article
} 


\section{Background}

Clinical reasoning is defined as the process by which information about a clinical problem is combined with the previous physicians' knowledge and experiences and used to manage a particular problem [1]. This process is an important factor in the physician's competence. Educators agree that clinical reasoning should be taught and tested in medical schools [2]. Attempts to assess clinical reasoning began in the 1970s [3]. The most popular test was the patient management problem(PMP) instrument developed at the University of Illinois [1]. This device is a test of clinical problem-solving skills in which each item begins with a clinical statement about the patient's problems on presentation. It is structured in stages, and the examinee is asked to make a decision that is appropriate to the situation $[4,5]$. Due to the long duration of PMP and its low reliability, other clinical reasoning tests were introduced, such as the key features (KF) test described in 1987 [6]. A $\mathrm{KF}$ is defined as a critical step in the resolution of a problem [7]. Testing with this instrument for the Canadian Qualifying Examination in medicine was shown to have an acceptable content validity and a reliability of 0.8 in 4 hours of examination time [8].

The script concordance test (SCT) is a clinical reasoning test used to assess reasoning in ambiguous situations [9]. This test is case-based, and items describe short scenarios followed by a series of questions presented in three parts. Part one contains a relevant diagnostic or management option, part two presents a new clinical finding and part three is a five-point Likert scale from -2 to +2 that indicates examinees' decisions [10]. The SCT is based on the principle that concordance can be measured between the examinees' answers and a panel of experts' judgments [11]. Research on the reliability and validity of SCT showed they are content valid, reliable and linearly related to experience [12-15], however more research is required to evaluate differential validity compared to multiple choice questions and predictive validity linked to clinical performance [16]

Another test of clinical reasoning is the set of clinical reasoning problems (CRP). In these items a scenario is presented and examinees are asked to nominate the two diagnoses they consider most likely, list the features of the case they think are important for the diagnosis, and indicate whether these features are positively or negatively predictive. Few studies was done on validity and reliability of CRPs but results showed an improved estimate of validity and reliability, especially proving the usefulness of CRPs as an indicator of the accuracy of the diagnostic reasoning [17].

Another assessment method is the comprehensive integrative puzzle (CIP). Items in this test are presented in the format of an extended matrix of rows and columns, in which examinees must insert the correct information in each cell. This test measures diagnostic thinking and clinical reasoning, However, this instrument seems to appeal more to students because of the fun in solving matching puzzles [18].

Research on clinical reasoning is scattered throughout medical education journals or publications in other field such as cognitive psychology and clinical psychology. Each of the tests described above was introduced in different studies, and to our knowledge different kinds of clinical reasoning tests have not been systematically studied or compared. The purpose of our study was to design a multi instrument for multiple roles approach in clinical reasoning field based on the theoretical framework, KF was used to measure data gathering, CRP was used to measure hypothesis formation, SCT and CIP were used to measure hypothesis evaluation and investigating the combined use of these tests in a single, nationwide, comprehensive, competitive examination for medical students known as the National Medical Science Olympiad. We examined the correlation between the total examination score and scores on each of four clinical reasoning tests. We also searched for a possible correlation between the total examination score and another measure of knowledge (grade point average).

\section{Methods}

The main aim of the Medical Science Olympiad in Iran is to test creative and critical thinking in medical students. The specific objectives of Olympiad were: Identifying scientifically talented individuals, Motivating and encouraging scientifically talented Individuals, Orienting extra-circular scientific activities, Generating scientific liveliness and morale, Interuniversity cultural exchanges, Encouraging to creative and critical thinking, Reinforcing health system goals and objectives, Encouraging team work, Encouraging interdisciplinary activities [19].

The first Olympiad, held in Isfahan in 2009, and the second in Shiraz in 2010, comprised a separate examination in each of three areas: basic science, clinical science and health system management. All currently enrolled medical students with a grade point average of $16 / 20$ (equivalent to a GPA of about 3.2 in the USA or a UK Class of about $60)$ or higher were eligible to register for the test. Then they prepared for the test by completing an intensive training course in the area of their choice at their own university. After this course enrollees were tested for critical thinking and reasoning skills at their university, and only those with the highest grades were then allowed to participate in the national Olympiad. Iran has 46 medical universities and each university is allowed to send only 3 students in each of the three areas to the Olympiad.

In the second Olympiad, 45 medical universities sent examinees for the areas of basic science and clinical 
science, and 44 medical universities sent examinees for the area of health system management. A total of 135 students took the test in basic science, 135 students were tested in clinical science, and 131 students took the test for the management area. In this study we analyzed the results only for the examination in the clinical science area. Only undergraduate students were allowed to participate in the Olympiad because of the importance of clinical reasoning skills in an early stage of their medical education and the need for efficient tools to assess it.

\section{Development of the clinical reasoning tests}

An expert committee with members from all Iranian medical schools was constituted and charged with developing a bank of test items in emergency medicine from all four clinical reasoning tests (i.e., KF, SCT CRP and CIP). The committee used the methodology described in previous publications [6-18]. Some examples of these tests are provided in additional file 1.

\section{Development of the Olympiad examination by the reference panel}

To prepare the examination to be used in the Olympiad, a total of 15 experts from different medical universities in Iran were chosen to constitute the reference panel. These experts comprised a broad sample of internists, general surgeons and emergency medicine specialists with different levels of experience and training, and were therefore considered to represent a normative sample of the reference population. Each member of the reference panel took each of the four tests and identified test items that were confusing or not relevant to emergency medicine. As a result, a few minor changes were made in the wording of some items. Then $20 \mathrm{KF}$ items, $20 \mathrm{SCT}$ items, $10 \mathrm{CRP}$ items and two $4 \times 6$ matrices from the CIP were chosen for inclusion in the full 2-day Olympiad examination. On the morning of the first day the $20 \mathrm{KF}$ items were completed, and in the afternoon the 10 CRP items were completed. On the morning of the second day the $20 \mathrm{SCT}$ items were completed, and in the afternoon the two CIP matrices were completed. Each of the four examination periods lasted 4 hours.

\section{Examinees}

The examinees in the second Olympiad were 135 undergraduate medical students from 45 medical schools in Iran, with grade point average if $16 / 20$ or higher. The length of medical education in Iran is 7 years.57.8 percent of participants were females and 42.2 percents were males. The mean year of study of participants was 6.1 years, the mean age of them was 24,3 years and the mean grade point average of them were 17.6 from 20 .

\section{Scoring process}

A group of 22 general practitioners and first-year residents were asked to complete all Olympiad examination items in their own time without using textbooks, web sites or personal consultations. General practitioners and first-year residents were recruited for this group because of their experience with a wide range of clinical problems encompassing all areas of emergency medicine practice. The scores obtained by these examinees were used as a standard reference [20].

\section{KF scoring}

To enhance the discriminating power of this score, we also calculated the efficiency score (partial credit score) [8].

\section{SCT scoring}

For high-stakes SCT examinations a reference group of more than 20 members is required [21]; as noted above, our reference group consisted of 22 physicians. Because of issues with aggregated scoring such as greater random error [16], we used average expert response weighted for distance and the correct answer on a five-point Likert scale. The mean response was considered the correct answer, and the weight for other responses was determined based on their credit and distance from the correct answer. With this scoring system the credit for the best answer was $100 \%$, and credit for other answers was calculated based on the percentage of reference panel examinees who chose that answer. We used the formula $1 /(1+x)$, where $x$ is defined as the distance from the correct answer (values of $x$ ranged from a minimum of 1 to a maximum of 4 ). This innovative scoring system was devised in the light of an analysis by Bland et al. [16] and consultation with a mathematician familiar with that research.

\section{CRP scoring}

The first and second diagnoses and diagnostic features chosen for each item by reference group examinees were input into a table, and the diagnoses and nominated features that were chosen by at least two thirds of the reference group were considered the correct answers.

\section{CIP scoring}

Examinees' scores were calculated from a matrix of answers given by the reference panel. For each of the 4 columns of cells in the matrix, 4 correct answers out of 4 (4/4) was scored as $100 \%, 3 / 4$ as $75 \%, 2 / 4$ as $50 \%$ and $1 / 4$ as $0 \%$. The grade for an entire matrix was considered the sum of the grades for all six rows and the grade for CIP exam was measured by the sum of two matrix grades.

\section{Total exam scores}

The total exam score was measured by the sum of 4 tests grade, therefore each test counts 25 percent of the 
total grade. The expert committee believed that 10 CRPs is similar to $20 \mathrm{KFs}$ or $20 \mathrm{SCT}$ because in CRP the students should choose two diagnoses and list the features of case based on these two diagnoses. In the CIP due to complexity of puzzles the expert committee considered two 6*4 puzzle similar to $20 \mathrm{KFs}$ or $20 \mathrm{CRPs}$. As we mentioned earlier the similar exam time was considered for each of the four tests(four hour for each tests).

\section{Analysis}

We measured item difficulty for each test, and determined the reliability of the scoring method for each test. The reliability of each test was calculated with Cronbach's alpha, considering each item individually and the combined reliability for all four clinical reasoning tests was calculated using variances of score in each test and total exam variance [22]. Item difficulty was determined with the method of Whitney and Sabers [23], and correlations between the total examination score and scores for each item were calculated with Pearson's correlation coefficient for each of the four clinical reasoning tests. The correlation between the total score and scores on each of the four tests was also calculated, along with the correlation between the total score on the Olympiad and the student's university course grade point average. We sought an informed consent from participants and ethical approval for our study from Olympiad clinical domain.

\section{Results}

The scores of each test in the Olympiad, total Olympiad score and maximum and minimum of each scor are shown in table 1 . The reliability of KF was .83, and this measure was .78 for SCT,.71 for CRP and .91 for CIP. The combined reliability for all four clinical reasoning tests was 0.91 .

Our findings for item difficulty level and item-total correlation are summarized for all $20 \mathrm{KF}$ items in Table 2, all 20 SCT items in Table 3, all 10 CRP items in Table 4, and the two $6 \times 4$ matrices in Table 5 . An item difficulty level between 0.2 and 0.8 is recommended [24] to differentiate between high- and low-achieving students, and the item-total correlations should be positive. In the KF test, item difficulty for all 20 items was between 0.52 and 0.79 . For the CRP test, all 10 items had a difficulty index between 0.40 and 0.69 . In the SCT (the most difficult of the four tests), item difficulty was between 0.25 and to
0.57 for 19 of the 20 items, but was 0.15 for one of the items. Item difficulty in the CIP test ranged from 0.39 to 0.90 .

All item-total correlations were positive. The correlations between each of the clinical reasoning test scores and the total examination score were high, although the highest correlations were seen for the KF and CIP tests (Table 6).

The correlation between scores on each clinical reasoning test and students' grade point average was low to intermediate for different tests (Table 7).

\section{Discussion}

The purpose of this study was to investigate the combined use of four different clinical reasoning tests (KF, SCT, CRP and CIP) in a high-stakes national examination designed to test clinical reasoning and decision-making skills in medical school undergraduates. Our results showed that the reliability of all four clinical reasoning tests was high. The most reliable tests were the CIP followed by the KF test, whereas the reliability of the SCT and CRP test was lower.

Different studies have reported varying reliabilities for these tests. The reliability for the KF test has been variously reported as 0.49 [25], 0.65 [26] and 0.80 in a 4hour examination [8]. Our partial credit scoring approach for this test led to more reliable results than in other studies. Although few studies have focused on the CRP, earlier reliability values ranged from 0.61 to 0.83 [17], which were similar to the reliability values we found. In the present study the reliability of the SCT was 0.78 . The psychometric properties of five scoring methods applied to the SCT were determined by Bland and et al. [16]. The reliability of these scoring methods ranged from 0.68 to 0.78 . Bland and colleagues reported that single-best-answer scoring with three answer choices produced results similar to aggregate scoring on a Likert-type scale, although they concluded that the optimal SCT scoring process is still debated [16]. In the present study the expert committee that chose the items for inclusion in the Olympiad examination believed that three answer choices increased the probability of choosing the answer by chance. The average expert response weighted for distance with our innovative formula and the correct answer from a five-point Likert scale showed acceptable reliability, although further research is

Table 1 Olympiad examination Scores

\begin{tabular}{ccccc}
\hline & $\begin{array}{c}\text { KF score } \\
\text { (from 500) }\end{array}$ & $\begin{array}{c}\text { SCT score } \\
\text { (from 500) }\end{array}$ & $\begin{array}{c}\text { CRP score } \\
\text { (from 500) }\end{array}$ & $\begin{array}{c}\text { CIP score } \\
\text { (from 500) }\end{array}$ \\
\hline Mean score \pm SD & $284.06 \pm 40.39$ & $217.66 \pm 40.30$ & $166.19 \pm 36.14$ & $258.58 \pm 84.84$ \\
\hline Maximum & 353 & 316 & 302 & 378 \\
\hline Minimum & 150 & 112 & 81 & $1060.84 \pm 201.56$ \\
\hline
\end{tabular}


Table 2 Item difficulty level and item-total correlations for the Key Features (KF) test

\begin{tabular}{lllllllllll}
\hline & KF1 & KF2 & KF3 & KF4 & KF5 & KF6 & KF7 & KF8 & KF9 & KF10 \\
\hline Item difficulty level & 0.52 & 0.66 & 0.66 & 0.76 & 0.77 & 0.69 & 0.69 & 0.79 & 0.71 & 0.71 \\
\hline Item-total correlation & 0.51 & 0.38 & 0.53 & 0.30 & 0.33 & 0.59 & 0.50 & 0.37 & 0.39 & 0.28 \\
\hline & KF11 & KF12 & KF13 & KF14 & KF15 & KF16 & KF17 & KF18 & KF19 & KF20 \\
\hline Item difficulty level & 0.70 & 0.74 & 0.72 & 0.66 & 0.78 & 0.57 & 0.64 & 0.61 & 0.76 & 0.53 \\
\hline Item-total correlation & 0.33 & 0.37 & 0.38 & 0.49 & 0.49 & 0.59 & 0.14 & 0.56 & 0.54 & 0.49 \\
\hline
\end{tabular}

Table 3 Item difficulty level and item-total correlations for the Script Concordance Test (SCT)

\begin{tabular}{|c|c|c|c|c|c|c|c|c|c|c|}
\hline & SC1 & $\mathrm{SC} 2$ & SC3 & SC4 & SC5 & SC6 & SC7 & SC8 & SC9 & SC10 \\
\hline Item difficulty level & 0.36 & 0.52 & 0.25 & 0.60 & 0.48 & 0.44 & 0.43 & 0.42 & 0.57 & 0.48 \\
\hline \multirow[t]{2}{*}{ Item-total correlation } & 0.41 & 0.46 & 0.32 & 0.45 & 0.50 & 0.37 & 0.52 & 0.48 & 0.33 & 0.41 \\
\hline & SC11 & $\mathrm{SC} 12$ & SC13 & SC14 & SC15 & SC16 & SC17 & SC18 & SC19 & SC20 \\
\hline Item difficulty level & 0.49 & 0.40 & 0.43 & 0.24 & 0.51 & 0.32 & 0.49 & 0.48 & 0.15 & 0.21 \\
\hline Item-total correlation & 0.54 & 0.41 & 0.30 & 0.19 & 0.30 & 0.34 & 0.28 & 0.41 & 0.12 & 0.09 \\
\hline
\end{tabular}

Table 4 Item difficulty level and item-total correlations for the Clinical Reasoning Problems (CRP) test

\begin{tabular}{llllllllllll}
\hline & CRP1 & CRP2 & CRP3 & CRP4 & CRP5 & CRP6 & CRP7 & CRP8 & CRP9 & CRP10 \\
\hline Item difficulty level & 0.57 & 0.51 & 0.69 & 0.54 & 0.40 & 0.56 & 0.43 & 0.64 & 0.49 & 0.53 \\
\hline Item-total correlation & 0.61 & 0.39 & 0.44 & 0.44 & 0.50 & 0.51 & 0.49 & 0.32 & 0.49 & 0.52 \\
\hline
\end{tabular}

Table 5 Item difficulty level and item-total correlations for the Comprehensive Integrative Puzzles (CIP) test

\begin{tabular}{llllllllll}
\hline & Q1 & Q2 & Q3 & Q4 & Q5 & Q6 & Q7 & Q8 & Q9 \\
\hline Item difficulty level & 0.90 & 0.56 & 0.56 & 0.59 & 0.69 & 0.56 & 0.39 & 0.55 & 0.57 \\
\hline Item-total correlation & 0.36 & 0.67 & 0.69 & 0.53 & 0.66 & 0.51 & 0.50 & 0.69 & 0.66 \\
\hline & $\mathbf{Q 1 0}$ & $\mathbf{Q 1 1}$ & $\mathbf{Q 1 2}$ & $\mathbf{Q 1 3}$ & $\mathbf{Q 1 4}$ & $\mathbf{Q 1 5}$ & $\mathbf{Q 1 6}$ & $\mathbf{Q 1 7}$ & $\mathbf{Q 1 8}$ \\
\hline Item difficulty level & 0.50 & 0.50 & 0.51 & 0.67 & 0.60 & 0.57 & 0.72 & 0.68 & 0.66 \\
\hline Item-total correlation & 0.55 & 0.67 & 0.59 & 0.77 & 0.64 & 0.66 & 0.78 & 0.77 & 0.80 \\
\hline
\end{tabular}

Table 6 Correlations between total Olympiad examination score and scores on each of the four clinical reasoning tests

\begin{tabular}{lllll}
\hline $\begin{array}{l}\text { Correlation } \\
\text { coefficient }\end{array}$ & $\begin{array}{l}\text { Key Features } \\
(\text { KF) }\end{array}$ & $\begin{array}{l}\text { Clinical Reasoning Problems } \\
\text { (CRP) }\end{array}$ & $\begin{array}{l}\text { Script Concordance Test } \\
\text { (SCT) }\end{array}$ & $\begin{array}{l}\text { Comprehensive Integrative Puzzle } \\
\text { (CIP) }\end{array}$ \\
\hline Total grade & 0.80 & 0.74 & 0.67 & 0.77 \\
\hline Significance $(P$ value) & $<0.001$ & $<0.001$ & $<0.001$ & $<0.001$ \\
\hline
\end{tabular}

Table 7 Correlations between grade point average and scores on each of the four clinical reasoning tests

\begin{tabular}{lllll}
\hline $\begin{array}{l}\text { Correlation } \\
\text { coefficient }\end{array}$ & $\begin{array}{l}\text { Key Features } \\
(\text { KF) }\end{array}$ & $\begin{array}{l}\text { Clinical Reasoning Problems } \\
\text { (CRP) }\end{array}$ & $\begin{array}{l}\text { Script Concordance Test } \\
\text { (SCT) }\end{array}$ & $\begin{array}{l}\text { Comprehensive Integrative Puzzle } \\
\text { (CIP) }\end{array}$ \\
\hline Grade point average & 0.26 & 0.27 & 0.13 & 0.18 \\
\hline Significance $(P$ value) & $<0.01$ & $<0.01$ & $>0.10$ & $<0.05$ \\
\hline
\end{tabular}


necessary to compare this method with previous scoring methods.

With regard to item difficulty, except for item number 19 on the SCT, all other test items had an acceptable level of difficulty between 0.20 to 0.80 . In terms of itemtotal correlations, all correlations were positive although for a few items this correlation was poor. In general, the findings for these correlations showed KF item 17 and SCT items 14, 19 and 20 were not able to discriminate effectively between high-achieving and low-achieving participants.

Our positive results, together with content validation of the tests before the Olympiad, enhanced the validity of the four-part, two-day examination. High correlation between each of the clinical reasoning tests and total Olympiad grade was an indicator for concurrent validity of these tests and also construct validity of the whole examination. However, the correlations between the clinical reasoning test results and grade point averages was low to intermediate, a finding that supports the idea that routine examination at medical universities in our setting measures students' factual knowledge more than their clinical reasoning skills.

Among the most important strengths of the present study is the large sample of examinees from all medical universities in Iran. Moreover, we used an expert panel of teachers from different medical universities to screen, select and adapt the items from all four tests that they felt were mostly likely to yield accurate results. The main limitation of our study was that examinees were strictly screened and selected from among the best students at each medical university. Olympiad scores cannot be viewed as generalizable to the whole population of medical students. This restriction of range may actually have enhanced the finding especially item difficulty level and correlations if all students had participated. Other limitations of our study were the facts that we did not compare different scoring methods, the penand-pencil format of the Olympiad, and the manual scoring of the Olympiad examinations. Some of these strengths and limitations are reported in results of the first Olympiad in Isfahan too [27]. This indicate the need to improve technical elements of the examination such as computerized administration and scoring. Future studies should be designed to validate our examination design and assessment methods.

\section{Conclusion}

We hope that the combination of clinical reasoning tests we used in a high-stakes national level examination for medical school undergraduates will provide evidence to support future actions aimed at enhancing the reliability of this exercise. We further hope that this report will help to raise the important issue of test reliability and motivate other universities and medical schools in other settings to examine their testing policies.

\section{Additional material}

Additional file 1: Appendix. A sample of clinical reasoning tests

\section{Acknowledgements}

We thank the Ministry of Health and Medical Education of Iran and the ViceChancellor for Educational and Students Affairs of Shiraz University of Medical Sciences for financial and scientific support for this project. We would like to express our gratitude to all the students who participated in the Olympiad and all of the medical university Education Development Center managers in Iran for coordinating the activities at the medical universities. We would like to give our special thanks to the staff of Shiraz Education Development Center. We express our appreciation to colleagues at Isfahan University of Medical Sciences and to the Olympiad Technical Committee, without whose guidance the second Olympiad would not have been possible. we thank K. Shashok for improving the use of English and the reporting in the manuscript, and Professor Cees P.M. van der Vleuten for reviewing an earlier version of the manuscript.

\section{Author details}

'Education Development and Research Center, Shiraz University of Medical Sciences, Shiraz, Iran. ${ }^{2}$ Emam Hosien Hospital Emergency Department, Shahid Beheshti University of Medical Sciences, Tehran, Iran. ${ }^{3}$ Internal Medicine Aliebneabitaleb Hospital, Zahedan University of Medical Sciences, Zahedan, Iran. ${ }^{4}$ Emam Reza Emergency Medicine Department, Mashhad University of Medical Sciences, Mashhad, Iran. ${ }^{5}$ Emergency Medicine Department, Baghiatallah Hospital, Baghiatallah University of Medical Sciences, Tehran, Iran. ${ }^{6}$ Department of Emergency Medicine, Guilan University of Medical Science, Rasht, Iran. 'Internal Medicine Department, Babol University of Medical Sciences, Babol, Iran. ${ }^{8}$ Pediatric General Surgery, Islamic Azad University, Mashhad Branch, Mashhad, Iran. ${ }^{9}$ Internal Medicine Department, Ahwaz Hospital, Ahwaz University of Medical Sciences, Ahwaz, Iran. ${ }^{10}$ Medical Humanities Research Group, Tehran, Institute for Humanities and Cultural Studies, Tehran, Iran. ${ }^{11}$ Firozgar Hospital of Internal Medicine, Tehran University of Medical Sciences, Tehran, Iran. ${ }^{12}$ Integrative Functional Gastroenterology Research Centers, Isfahan University of Medical Sciences, Isfahan, and Head of the Olympiad Technical Committee, Ministry of Health and Medical Education, Tehran, Iran. ${ }^{13}$ Recherche et Développement, Centre de Pédagogie Appliquée aux Sciences de la Santé, Montreal University, Montreal, Canada.

\section{Authors' contributions}

MA-MM-JK coordinated the team, collected the data, contributed to data analysis and interpretation, and drafted the manuscript. AM - KSA- PA contributed to the conception of clinical reasoning battery in Olympiad, contributed to design of the study and supervised the whole study. HAAAD-NAM-EP-HJ-VMK-HV-MARS-RA-BO-AS-MD-MM contributed to the design of the various tests and involved in scoring them. BC commented on the draft at all stages. All authors read and approved the final manuscript.

\section{Competing interests}

The authors declare that they have no competing interests.

Received: 16 May 2011 Accepted: 17 October 2011

Published: 17 October 2011

\section{References}

1. Newble D, Norma G, Van der Vulten CP: Assessing clinical reasoning in. In Clinical reasoning in health professions Edited by: Jones JHM , 2156-188.

2. Van der Vulten CPM, Newble DI: How can we test clinical reasoning? Lancet 1995, 345:1032-1034.

3. Norman G: Research in clinical reasoning: Past history and current trends. Medical Education 2005, 39:418-427. 
4. Harden RM: Preparation and presentation of patient management problems (PMPS). Medical Education 1983, 17(4):255-276.

5. Marshall JR, Fabb WE: The construction of patient management problems. Medical Education 1981, 15(2):126-135.

6. Bordage G, Page G: An alternate approach to PMPS, the key feature concept. In Further developments in assessing clinical competent Montreal. Edited by: Hart I, Harden R. Can Heal Publications; 1987:57-75.

7. Farmer EA, Page G: A practical guide to assessing clinical decision making skills using the key feature approach. Medical Education 2005, 39:1188-1194

8. Page G, Bordage G: The medical council of Canada's key feature project: a more valid written examination of clinical decision making sills. Acad Med 1995, 70:104-110.

9. Fournier JP, Demeester A, Charlin B: Script Concordance tests: guideline for constriction. BMC medical informatics and decision making 2008, 8(18):1-7.

10. Charlin B, Boshuizen HPA, Custers EJFM, Feltovich PJ: Scripts and clinical reasoning. Medical Education 2007, 41:1179-1185.

11. Charlin B, Vander ulten CPM: Standardized assessment of reasoning in context of uncertainty. The script concordance test approach. Evaluation and the Health Profession 2004, 27:304-319.

12. Brailovsky C, Charlin B, Beausoleil S, Cote S, Van der Vleuten C: Measurement of clinical reflective capacity early in training as a predictor of clinical reasoning performance at the end of residency: an exploratory study on the script concordance test. Med Educ 2001, 35:430-6.

13. Charlin B, Brailovsky CA, Brazeau-Lamontaque L, Samson L, Leduc C: Script questionnaires: their use for assessment of diagnostic knowledge in radiology. Med Teach 1998, 20:567-71.

14. Charlin B, Brailovsky CA, Leduc C, Blouin D: The diagnostic script questionnaire: a new tool to assess a specific dimension of clinical competence. Adv Health Sci Educ 1998, 3:51-8.

15. Charlin B, Brailovsky C, Roy L, Goulet F, van der Vleuten C: The script concordance test: a tool to assess the reflective clinician. Teach Learn Med 2000, 12:189-95.

16. Bland AC, Clarence D, Kreiter CD, Gordon JA: The psychometric properties of five scoring methods applied to the script concordance test. Academic Medicine 2005, 80(4):395-9.

17. Groves M, Scott I, Alexander H: Assessing clinical reasoning: a method to monitor its development in a PBL curriculum. Medical Teacher 2002, 24(5):507-515.

18. Ber R: The CIP (comprehensive Integrative puzzle) assessment method. Medical Teacher 2003, 25(2):171-176.

19. Amini M, Kojuri J, Karimian Z, Lotfi F, Moghadami M, Dehghani MR, et al: Talents for future: Report of the second national medical science Olympiad in Islamic republic of Iran. Iran Red Crescent Med J 2011, 13(6):377-381.

20. Norcini J, Shea JA, Day SC: The use of the aggregated scoring for a recertification examination. Eval Health Prof 1990, 13:241-251.

21. Gagnon R, Charlin B, Coletti M, Sauve E, Van der vulten CPM: Assessment in the context of uncertainty: How many members are needed on the panel of reference of a script concordance test? Medical Education 2005 39:284-291.

22. Carmines EG, Zeller RA: Relability and validity assessment. Sage 1997.

23. Whitney DR, Sabers DL: Improving essay examinations III: Use of item analysis. lowa City: University of lowa, University Evaluation and Examination Service (Technical Bulletin No. 11); 1970.

24. Bortz J, Do Ring N: Forschungs methoden und Evaluation. Berlin, Springer; 32002.

25. Hatala R, Norman G: Adapting the key features examination for a clinical clerkship. Medical Education 2002, 36:160-165.

26. Fischer MR, Kopp V, Holzer M, Ruderich F, Junger J: A modified electronic key feature examination for undergraduate medical students: validation threats and opportunities. Medical Teacher 2005, 27(5):450-455.

27. Monajemi A, Adibi P, Soltani Arabshahi K, Arbabi F, Akbari R, Custers E, HadadgaR A, Hadizadeh F, Changiz T: The battery for assessment of clinical reasoning in the Olympiad for medical sciences students. Iranian Journal of Medical Education 2010, 10(5):1056-1067. doi:10.1186/1756-0500-4-418

Cite this article as: Amini et al:: An innovative method to assess clinical reasoning skills: Clinical reasoning tests in the second national medical science Olympiad in Iran. BMC Research Notes 2011 4:418.

\section{Submit your next manuscript to BioMed Central and take full advantage of:}

- Convenient online submission

- Thorough peer review

- No space constraints or color figure charges

- Immediate publication on acceptance

- Inclusion in PubMed, CAS, Scopus and Google Scholar

- Research which is freely available for redistribution

Submit your manuscript at www.biomedcentral.com/submit
C Biomed Central 